\title{
EDITORIAL
}

\section{EVOLUCIÓN DE LA MORTALIDAD EN POBLACIÓN RECLUSA TRAS LA INTRODUCCIÓN DEL TRATAMIENTO ANTIRRETROVIRAL DE GRAN ACTIVIDAD (TARGA)}

\author{
Andrés Marco \\ Programa VIH/SIDA y VHC. Centre Penitenciari d'Homes de Barcelona. Departament de Justícia. Gene- \\ ralitat de Catalunya
}

Hay aproximadamente 10 millones de personas encarceladas en el mundo y se calcula que el número de presos ha aumentado en más de un millón en la última déca$\mathrm{da}^{1}$. En la población reclusa los problemas de salud más frecuentes son los trastornos mentales, el abuso de alcohol, el consumo de drogas ilegales y las infecciones por virus hepatotropos, por el VIH y por el bacilo de la tuberculosis, así como las conductas violentas, autolesivas o heteroagre$\operatorname{sivas}^{2-6}$. A pesar de que estas circunstancias harían pensar lo contrario, un reciente estudio realizado en Georgia, USA ${ }^{7}$, estima que la tasa global de mortalidad en prisión es baja aunque hay, como también han citado otros estudios ${ }^{3,4,8-15}$, una elevada mortalidad por causas de origen violento (suicidio, agresiones, etc.). Sin embargo, en las prisiones españolas, en 2007, la tasa de mortalidad por causas violentas fue de $1,27 \%$, inferior a la producida por enfermedad (2,28\%o), que suponía el 63,9\% de los casos de muerte registrados ${ }^{16}$. Y debe tenerse presente, además, que la tasa de mortalidad por enfermedad hace referencia exclusivamente a las muertes ocurridas durante el encarcelamiento y no incluye, por tanto, los éxitus de personas excarceladas por libertad anticipada debida al diagnóstico de enfermedad grave con mal pronóstico vital.
Desde finales de los años ochenta y hasta la aparición del TARGA en 1996, en los países occidentales, la principal causa de muerte en población reclusa fue el $\mathrm{Sida}^{7,17-21}$, ya que muchas de las personas internadas eran usuarias de drogas endovenosas y estaban infectadas por el VIH. Desde entonces, la prevalencia de esta infección ha pasado del $40 \%$ en aquella época hasta aproximadamente el $10 \%$ actual $^{22}$. Este notable descenso de la infección, junto a la elevada efectividad del TARGA, ha ocasionado una disminución de la morbilidad por VIH/SIDA ${ }^{23,24}$, una disminución de los ingresos hospitalarios ${ }^{24} \mathrm{y}$ una mayor supervivencia de los sujetos infectados por el VIH1 ${ }^{7,25-27}$. Dado el descenso de las muertes por Sida ocurrido en los últimos años, cabe preguntarse cuáles son ahora las principales causas de muerte en la población internada en prisión.

Para conocer la situación actual disponemos de algunos estudios de cohortes que han sido realizados durante la última déca$\mathrm{da}^{4,7,11,21,27,28}$ en Estados Unidos, Canadá, Australia, Reino Unido y España. Es de destacar que dos de estos trabajos han sido efectuados en nuestro país, ambos por el mismo grupo investigador, en el mismo centro de trabajo y con la ventaja de que uno se efectuó en el periodo 1994-2004 ${ }^{21}$ y otro, publicado en este número de la Revista Española de 
Salud Pública ${ }^{28}$, ha ampliado el estudio hasta el año 2009, lo que permite comprobar las principales causas de muerte por periodos temporales, la tendencia de la mortalidad y la SMR o razón de mortalidad estandarizada (muertes observadas/esperadas). Debe recalcarse que estos dos trabajos se han efectuado en una única prisión, que es además de mediano tamaño, y por consiguiente puede ser arriesgado extrapolar sus resultados al conjunto del territorio español, como los propios autores reconocen ${ }^{28}$. Pero aún teniendo en cuenta esta limitación, en España, a la vista de las investigaciones efectuadas y del análisis de los registros oficiales de mortalidad de las Administraciones Penitenciarias, puede afirmarse:

a) que el número de defunciones ocurridas en prisión se ha reducido en los últimos años considerablemente, hasta el extremo de que la tasa cruda de mortalidad, es decir sin apareamientos por grupos de enfermedad, edad o sexo, que antaño era muy superior a la de la población general, actualmente es menor que la de la población no encarcelada. En concreto, en el trabajo de García-Guerrero et al. la tasa cruda global de mortalidad fue de $6,182 \%$ durante el periodo estudiado, oscilando entre el 12,605\% en 1997 y el 1,757\% en 2003, mientras que la tasa de mortalidad de la población general española en el año 2009 fue de $8,343 \%{ }^{29}$.

b) que la disminución de la tasa de mortalidad se ha producido fundamentalmente al mejorar la supervivencia de las personas infectadas por el VIH, y

c) que la mortalidad de origen no violento y por enfermedad ajena al Sida es la más frecuente en el momento presente.

En cuanto a las actuales causas de muerte, últimamente se ha observado en nuestro país, así como en otros países, un aumento de la mortalidad de origen cardiovascular $4,8,9,27,28,30$ y también de tipo neoplásico ${ }^{4,31}$, que son ahora las causas de defunción más habituales.
Esto probablemente se deba a factores como el hábito tabáquico, el consumo de cocaína y/o alcohol, o el envejecimiento de la población penitenciaria ocurrido en los últimos años, aunque la mayor supervivencia de los sujetos infectados por el VIH también puede influir, ya que se ha demostrado que la morbimortalidad cardiovascular y neoplásica es mayor en esta población ${ }^{32-35}$. Además es muy probable que la mortalidad de origen cardiovascular y neoplásico todavía se incremente más en el futuro, si continúa aumentando la edad de la población reclusa y se reduce la prevalencia de algunas infecciones que hasta hace poco han tenido un enorme peso en la morbi-mortalidad de los presos.

Ante el previsible aumento de la mortalidad cardiovascular y neoplásica en la población penitenciaria, ¿qué puede hacerse? Probablemente sea necesario diseñar e implementar estrategias preventivas (para dejar de fumar, para promover el ejercicio aeróbico, para evitar el sobrepeso y la obesidad, etc.) que tengan como objeto reducir causas de mortalidad prematura que habían quedado en segundo término por la importancia de la epidemia del VIH/Sida en prisión. Y todo ello sin olvidar los programas que han mostrado un notable éxito (de reducción de daños asociados al consumo de drogas, de prevención de suicidios, de prevención y control de enfermedades infecciosas...) y que continúan siendo necesarios en este grupo de población.

\section{BIBLIOGRAFÍA}

1. Fazel S, Baillargeon J. The health of prisoners. Lancet. 2011; 377:956-65.

2. Fagan TJ, Cox J, Helfand SJ, Aufderheide D. Selfinjurious behavior in correctional settings. J Correct Health Care. 2010; 16: 48-66.

3. Fruehwald S, Frottier P, Matschnig T, Eher R. The relevance of suicidad behaviour in jail and prison suïcides. Eur Psychiatry 2003; 18: 161-5.

4. Wobeser WL, Datema J, Bechard B, Ford P. Causes of death among people in custody in Ontario, 1990-1999. CMAJ 2002;167:1109-13. 
5. Kim S, Ting A, Puisis M, Rodriguez S, Benson R, Mennella C, et al. Deaths in the Cook County jail: 10year report, 1995-2004. J Urban Health 2007; 84:70-84.

6. Bedoya A, Martínez-Carpio PA, Humet V, Leal MJ, Lleopart N. Incidencia del suicidio en las prisiones de Cataluña: analisis descriptivo y comparado. Rev Esp Sanid Penit. 2009; 11: 37-41.

7.Spaulding AC, Seals RM, McCallum VA, Perez SD, Brzozowski AK, Steenland NK. Prisoner survival inside and outside of the institution: implications for healthcare planning. Am J Epidemiol. 2011;173:479-87.

8. Salive ME, Smith GS, Brewer TF. Death in prison: changing mortality patterns among male prisoners in Maryland, 1979-87. Am J Public Health. 1990; 80:147980.

9. Chistiansen WF, Gregersen M. Deaths among inmates in the institutions of the Prison Service. Deaths in prisons, county jails etc. Ugeskr Laeger. 1999; 161:1410-4.

10. Novick LF, Remmlinger E. A study of 128 deaths in New York City correctional facilities (1971-1976): implications for prisoner health care. Med Care. 1978; 16:749-56.

11. Kariminia A, Law MG, Butler TG, Corben SP, Levy MH, Kaldor JM, et al. Factors associated with mortality in a cohort of Australian prisoners. Eur J Epidemiol. 2007; 22: 417-28.

12. Fazel S, Benning R. Suicides in female prisoners in England and Wales, 1978-2004. Br J Psychiatry 2009; 194:183-4.

13. Black DW, Gunter T, Loveless P, Allen J, Sieleni B. Antisocial personality disorder in incarcerated offenders: Psychiatric comorbidity and quality of life. Ann Clin Psychiatry. 2010;22:113-20.

14. Baillargeon J, Penn JV, Thomas CR, Temple JR, Baillargeon G, Murray OJ. Psychiatric disorders and suicide in the nation's largest state prison system. J Am Acad Psychiatry Law. 2009; 37:188-93.

15. Camilleri P, McArthur M. Suicidal behaviour in prisons: learning from Australian and international experiences. Int J Law Psychiatry. 2008; 31:297-307.

16. Secretaría General de Instituciones Penitenciarias. Coordinación de Sanidad Penitenciaria. Área de Salud Pública. Mortalidad en Instituciones Penitenciarias 2007. Bol Epidemiol Instit Penit. 2010; 14: 13-16.

17. Caylà JA, Marco A, Bedoya A, Guerrero R, García J, Martín V, et al. Differential characteristics of AIDS patients with a history of imprisonment. Int J Epidemiol. 1995; 24: 1188-96.

18. Anonymous. AIDS death rate for inmates falls in U.S. prisons, jails. AIDS Policy Law. 2005; 20:5

19. Morse DL, Truman BI, Hanrahan JP, Mikl J, Broaddus RK, Maguire BH, et al. AIDS behind bars. Epidemiology of New York State prison inmate cases, 19801988. N Y State J Med. 1990; 90:133-8.

20. Amankwaa AA. Causes of death in Florida prisons: the dominance of AIDS. Am J Public Health. 1995; $85: 1710-1$.

21. Vera-Remartínez EJ, Planelles-Ramos MV, GarcíaGuerrero J. Tendencia de la tasa de mortalidad en una prisión española (1994-2004). Rev Esp Salud Pública. 2005; 79: 673-82.

22. Marco A, Saíz de la Hoya P, García-Guerrero J, Rivera-Jiménez AJ, Vasallo L, Moreno-Moreno R, et al. Estudio multicéntrico de prevalencia de infección por VIH y factores asociados en presos espanyoles. Enferm Infecc Microbiol Clin. 2009; 27:16.

23. García-Guerrero J, Vera EJ, González-Morán F. Morbimortalidad hospitalaria en presos $\mathrm{VIH}+$ : diez años de cambiios. Rev Esp Sanid Penit. 2005; 7:14-22.

24. Arroyo JM. Public health gains from health in prisons in Spain. Public Health. 2010; 124:629-31.

25. Baillargeon J, Borucki M, Williamson J, Dunn K. Determinants of HIV-related survival among Texas prison inmates. AIDS Patient Care STDS. 1999;13:355-61.

26. Centers for Disease Control and Prevention (CDC). Decrease in AIDS-related mortality in a state correctional system--New York, 1995-1998. MMWR Morb Mortal Wkly Rep. 1999; 47: 1115-7.

27. Fazel S, Benning R. Natural deaths in male prisons: a 20-year mortality study. Eur J Public Health. 2006; 16: 441-4.

28. García-Guerrero J, Vera EJ, Planelles MV. Causas y tendencia de la mortalidad en una prisión española (1994-2009). Rev Esp Salud Publica. 2011; 247-57.

29. Instituto Nacional de Estadística. Indicadores demográficos básicos. Tasa Bruta de Mortalidad calculada para el año 2009 . Disponible en: http://www.ine.es/jaxi/tabla.do?per=12\&type=db\&divi $=$ IDB\&idtab=32. (Citado el 28/12/2010).

30. Roessler B, Fleischhackl R, Fleischhackl S, Singer F, Mittlboeck M, Fachberger J, et al. Death in correctional 
facilities: opportunities for automated external defibrillation. Resucitation. 2007;73:389-93.

31. Mathew P, Elting L, Cooksley C, Owen S, Lin J. Cancer in an incarcerated population. Cancer. 2005;104: 2197-204.

32. Law M, Friis-Møller N, Weber R, Reiss P, Thiebaut $\mathrm{R}$, Kirk O, et al. Modelling the 3-year risk of myocardial infarction among participants in the Data Collection on Adverse Events of Anti-HIV Drugs (DAD) study. HIV Med. 2003; 4:1-10.

33. Triant VA, Lee H, Hadigan C, Grinspoon SK. Increased acute myocardial infarction rates and cardiovascular risk factors among patients with human immunodeficiency virus disease. J Clin Endocrinol Metab. 20071; 92: 2506-12.

34. Cheung MC, Patanowitz L, Dezube BJ. AIDS-related malignancies: emerging challenges in the era of highly active antiretroviral therapy. Oncologist. 2005; 10:412-26.

35. Dauby N, De Wit S, Delforge M, Necsoi VC, Clumeck N.Characteristics of non-AIDS-defining malignancies in the HAART era: a clinico-epidemiological study. J Int AIDS. Soc 2011; 14:16 